\title{
Development of a Biomarker-Based Scoring System Predicting Early Recurrence of Resectable Pancreatic Duct Adenocarcinoma
}

\author{
Keinosuke Ishido, MD, PhD (1) , Norihisa Kimura, MD, PhD, Taiichi Wakiya, MD, PhD, \\ Hayato Nagase, MD, PhD, Yutaro Hara, MD, Taishu Kanda, MD, Hiroaki Fujita, MD, and \\ Kenichi Hakamada, MD, PhD
}

Department of Gastroenterological Surgery, Hirosaki University Graduate School of Medicine, Hirosaki, Japan

\begin{abstract}
Background. Resectable pancreatic ductal adenocarcinoma (R-PDAC) often recurs early after radical resection, which is associated with poor prognosis. Predicting early recurrence preoperatively is useful for determining the optimal treatment.

Patients and methods. One hundred and seventy-eight patients diagnosed with R-PDAC on computed tomography (CT) imaging and undergoing radical resection at Hirosaki University Hospital from 2005 to 2019 were retrospectively analyzed. Patients with recurrence within 6 months after resection formed the early recurrence (ER) group, while other patients constituted the non-early recurrence (non-ER) group. Early recurrence prediction score (ERP score) was developed using preoperative parameters.

Results. ER was observed in 45 patients (25.3\%). The ER group had significantly higher preoperative CA19-9 $(p=0.03)$, serum SPan-1 $(p=0.006)$, and CT tumor diameter $(p=0.01)$ compared with the non-ER group. The receiver operating characteristic (ROC) curve analysis identified cutoff values for CA19-9 (133 U/mL), SPan-1 $(78.2 \mathrm{U} / \mathrm{mL})$, and preoperative tumor diameter $(23 \mathrm{~mm})$. When the parameter exceeded the cutoff level, 1 point was given, and the total score of the three factors was defined as the ERP score. The group with an ERP score of 3 had postoperative recurrence-free survival (RFS) of 5.5 months (95\% CI 3.02-7.98). Multivariate analysis for ER-related
\end{abstract}

(C) The Author(s) 2021

First Received: 1 April 2021

Accepted: 11 September 2021;

Published Online: 4 October 2021

K. Ishido, MD, PhD

e-mail: k-ishido@hirosaki-u.ac.jp perioperative and surgical factors identified ERP score of 3 [odds ratio (OR) 4.63 (95\% CI 1.82-11.78), $p=0.0013$ ] and R1 resection [OR 3.20 (95\% CI $1.01-10.17)$, $p=0.049]$ as independent predictors of ER.

Conclusions. For R-PDAC, ER could be predicted by the scoring system using preoperative serum CA19-9 and SPan-1 levels and CT tumor diameter, which may have great significance in identifying patients with poor prognoses and avoiding unnecessary surgery.

Keywords Pancreatic ductal adenocarcinoma .

Resectable $\cdot$ Early recurrence $\cdot$ CA19-9 $\cdot$ SPan-1

Pancreatic ductal adenocarcinoma (PDAC) is a refractory disease with very poor prognosis. It is currently the fourth most common cause of cancer-related mortality in the USA, and has a 5-year survival rate of less than $10 \% .{ }^{1}$ Based on the current incidence of PDAC, it is expected to become the second leading cause of cancer-related deaths in Europe and the USA by $2030 .{ }^{2,3}$ Although radical resection is considered to be a curative treatment, the 5-year survival rate after curative resection is only $15-20 \%{ }^{4,5}$

When PDAC is diagnosed as resectable on computed tomography (CT), better survival, with 5-year survival of around $40 \%$, can be expected by promptly introducing adjuvant chemotherapy after radical resection. ${ }^{6-8}$ However, distant metastases are often detected early after resection, ${ }^{9,10}$ and some patients have to discontinue adjuvant chemotherapy. Therefore, the question of how to control such a malignant behavior has become a big challenge, even for patients with resectable PDAC. ${ }^{11}$

Previous studies investigated factors related to the uncontrollable behavior of PDAC. ${ }^{12,13}$ In particular, serum CA19-9 level is known to correlate with postoperative prognosis, and its association with early postoperative 
recurrence has also attracted attention. ${ }^{14,15}$ Preoperative serum CA19-9 level has been reported to be an independent factor that determines early postoperative recurrence, and it has been perceived as an indicator of poor postoperative prognosis. ${ }^{16-18}$ Other studies addressed the association between tumor immunonutrient factors, such as neutrophil-to-lymphocyte ratio (NLR) and modified Glasgow Prognostic Score (mGPS), and early postoperative recurrence. ${ }^{19,20}$ The significance of neoadjuvant therapy (NAT) for such difficult-to-control PDACs has been discussed. $^{21,22}$ The purpose of NAT is to achieve more reliable R0 resection and control latent distant metastasis preoperatively, which is expected to have a further prognostic effect on resectable PDAC. ${ }^{23}$ However, it has been also pointed out that the tumor may progress during the NAT period, and the surgical tolerance may decrease due to adverse events of NAT. A phase 2 randomized controlled trial comparing the contribution of perioperative FOFIRINOX and gemcitabine/nab-paclitaxel to survival in patients with R-PDAC was reported. ${ }^{24,25}$ According to the results, the 2-year survival rate, the primary endpoint, was $43 \%$ in the FOFIRINOX arm and $46.9 \%$ in the gemcitabine/nab-paclitaxel arm, with no significant difference between the groups. Furthermore, neither arm met the prespecified threshold of a 2-year overall survival rate of $58 \%$ based on historical data from adjuvant trials in R-PDAC. Therefore, this phase 2 trial did not have the expected outcome. Additionally, $75 \%$ of eligible patients were able to complete preoperative chemotherapy and undergo surgery, whereas the remaining patients were unable to undergo surgery because of disease progression or adverse events from preoperative chemotherapy. Thus, it is not well established whether NAT should be given to patients with resectable PDAC. It may be suggested that NAT is not appropriate for all cases of resectable PDAC. Hence, resectable PDAC should be further classified depending on the malignant potential of the tumor, and an appropriate treatment strategy should be chosen accordingly. In this context, pretreatment prediction of early recurrence is essential for treatment selection and improving the prognosis of resectable PDAC.

The aim of this study was to establish a prognostic scoring system based on preoperative biomarkers to identify early recurrence within 6 months after radical resection for resectable PDAC.

\section{PATIENTS AND METHODS}

\section{Ethics}

This retrospective study was approved by the Ethics Committee of the Graduate School of Medicine, Hirosaki
University. We obtained informed consent from all patients with regard to analyzing the data.

\section{Patients}

From January 2005 to December 2019, 200 patients were diagnosed with resectable PDAC using multi-detector row computed tomography (MDCT) at Hirosaki University Hospital. Preoperative MDCT was performed in all patients, and contrast-enhanced magnetic resonance imaging (MRI) and positron emission tomography-computed tomography (PET-CT) were performed as ancillary diagnostic imaging. When the diagnostic images were not enough to diagnose with PDAC, endoscopic ultrasoundguided fine needle aspiration (EUS-FNA) was applied to confirm the diagnosis. Among these 200 patients, we excluded 22 patients (2 individuals in whom only exploratory laparotomy was performed due to distant metastases, 2 individuals in whom palliative surgery was performed, 7 patients in whom radical surgery could not be obtained, and 11 patients in whom preoperative chemotherapy was performed). Finally, 178 cases were analyzed. Among these 178 patients, 45 patients with recurrence occurring within 6 months after radical surgery were included in the early recurrence group (ER group), whereas 133 patients in whom recurrence did not occur within 6 months constituted the non-early recurrence group (non-ER group). We compared the clinicopathological parameters between the two groups.

\section{Definition of Resectable PDAC}

Classification of resectability was performed in accordance with the National Comprehensive Cancer Network guidelines version $12020 .{ }^{26}$ Tumors meeting the following criteria were defined as resectable PDAC: (1) the tumor does not contact the major arteries (the celiac trunk, superior mesenteric artery, and common hepatic artery); (2) the tumor has no contact with the portal vein and superior mesenteric vein, or the contact is less than half of the circumference of the vessel wall, and it is not accompanied by venous contouring; (3) no distant metastasis was confirmed.

\section{Postoperative Complications}

Postoperative complications were evaluated based on the classification reported by Clavien et al. ${ }^{27}$ Postoperative pancreatic fistula was defined as grade $\mathrm{B}$ and grade $\mathrm{C}$ based on the grading of the International Study Group of Pancreatic Fistula. $^{28}$ 


\section{Pathological Evaluation}

Pathological evaluation of surgical specimens was based on the tumor-node-metastasis (TNM) classification system of malignant tumors by the International Union Against Cancer (UICC, 8th edition). ${ }^{29}$ Evaluation of the excised margin was performed as follows: if there were no tumor cells on the pancreatic resection margin, nerve plexus dissection margin, portal vein dissection surface, posterior dissection surface, and bile duct dissection margin, it was evaluated as an $\mathrm{R} 0$ resection. In contrast, if the tumor cells were recognized on these margins, the resection was evaluated as an R1 resection. In all cases, rapid intraoperative pathological diagnosis of the pancreatic resection margin was conducted, and additional resection was performed when tumor cells were still present on the stump surface.

\section{Postoperative Adjuvant Chemotherapy}

Postoperative adjuvant chemotherapy was introduced within 12 weeks after surgery and continued for 6 months. The chemotherapy regimen included gemcitabine (GEM) monotherapy, S-1 monotherapy, GEM and S-1 combination therapy, or GEM and nanoparticle albumin boundpaclitaxel combination therapy. No postoperative radiation was given in any of the patients. After the operation, blood examination including tumor markers (CA19-9, SPan-1, and DUPAN-2) every 4 weeks and CT examination every 3 months were performed to screen for postoperative recurrence. If the assessment of recurrence was uncertain, contrast-enhanced MRI or PET-CT was used as an adjunct method.

\section{Prediction Scoring System for Early Recurrence}

Univariate analysis with preoperative factors was performed to identify the factors associated with early recurrence. For the identified factors, the cutoff values for predicting early recurrence were estimated using the ROC curve analysis. For the factors exceeding the cutoff value, 1 point was added. The total score of the identified factors was taken as the early recurrence prediction score $(0$ point, 46 cases; 1 point, 64 cases; 2 points, 37 cases; 3 points, 31 cases).

\section{Statistical Analysis}

Categorical variables were compared between ER and non-ER groups with the chi-squared test or Fisher's exact test. Variables with $p<0.05$ were considered significant. Univariate and multivariate analyses of perioperative factors associated with early recurrence were conducted using logistic regression to identify independent factors related to early recurrence. The analysis of prognosis was based on the Kaplan-Meier curves. Log-rank test was used for comparison of progression-free survival and OS by early recurrence prediction scores, and $p<0.05$ indicated a significant difference. Statistical evaluation was performed using IBM SPSS statistics version 25 for Windows.

TABLE 1 Patient characteristics

\begin{tabular}{|c|c|}
\hline \multicolumn{2}{|l|}{ Parameter } \\
\hline Number of patients & 178 \\
\hline Age (years) & $70(49-85)$ \\
\hline Gender (female/male) & $86 / 92$ \\
\hline BMI & $22.3(14.1-36.8)$ \\
\hline ASA-PS $(1 / 2 / 3 / 4<)$ & $26 / 133 / 19 / 0$ \\
\hline Diabetes mellitus & $55(30.9 \%)$ \\
\hline Chronic pancreatitis & $7(3.9 \%)$ \\
\hline Tumor location $(\mathrm{Ph} / \mathrm{Pbt})$ & $118 / 60$ \\
\hline Diameter of the pancreatic tumor on CT (mm) & $25(8-59)$ \\
\hline Preoperative CA19-9 (U/mL) & $69(20-217)$ \\
\hline Preoperative SPAN-1 (U/mL) & $51.3(18.9-119.7)$ \\
\hline Preoperative DUPAN-2 (U/mL) & $99.5(27.8-412.8)$ \\
\hline \multicolumn{2}{|l|}{ Operative procedures } \\
\hline (PD/DP/TP) & $108 / 60 / 10$ \\
\hline Intraoperative transfusion & $32(18.0 \%)$ \\
\hline Lymph node metastasis & $108(60.7 \%)$ \\
\hline $\mathrm{R} 0$ resection rate & $159(89.3 \%)$ \\
\hline Overall morbidity (Clavien-Dindo > II) & $33(18.5 \%)$ \\
\hline 90-day mortality & $0(0.0 \%)$ \\
\hline Induction rate of adjuvant chemotherapy & $142(79.8 \%)$ \\
\hline Median recurrence-free survival time (months) & $13.3(10.7-15.9)$ \\
\hline Median survival time (months) & $30.6(25.5-35.7)$ \\
\hline Postoperative recurrence within 6 months & $45(25.2 \%)$ \\
\hline \multicolumn{2}{|l|}{ Recurrence site } \\
\hline Liver & $26(57.8 \%)$ \\
\hline Lung & $2(4.4 \%)$ \\
\hline Peritoneum & $14(31.1 \%)$ \\
\hline Distant lymph node & $10(22.2 \%)$ \\
\hline Locoregional site & $12(26.7 \%)$ \\
\hline
\end{tabular}

Age age at diagnosis, BMI body mass index, ASA-PS American Society of Anesthesiologists physical status, CA19-9 carbohydrate antigen 19-9, Span-1 s-pancreas-1 antigen, DUPAN-2 Duke pancreas2 antigen, $P D$ pancreaticoduodenectomy, $D P$ distal pancreatectomy, $T P$ total pancreatectomy 


\section{RESULTS}

\section{Patients' Background}

The background information of 178 patients with resectable PDAC is presented in Table 1 . The median age was 70 years, and the age range was $49-85$ years. There were 86 women and 92 men. As comorbidities, diabetes mellitus was found in 55 cases (30.9\%) and chronic pancreatitis in 7 cases (3.9\%). The location of PDAC was most often in the head of the pancreas (118 cases), followed by the body and tail of the pancreas in 60 cases. The median values of preoperative serum carbohydrate antigen (CA)199, serum s-Pancreas-1 antigen (SPan-1), and Duke Pancreas-2 Antigen (DUPAN-2) were $69 \mathrm{U} / \mathrm{mL}$ [interquartile range (IQR) $\quad 20-217 \mathrm{U} / \mathrm{mL}], \quad 51.3 \mathrm{U} / \mathrm{mL} \quad$ (IQR 18.9-119.7 U/mL), and $99.5 \mathrm{U} / \mathrm{mL}$ (IQR 27.8-412.8 U/ $\mathrm{mL}$ ), respectively. The performed surgical procedures included pancreaticoduodenectomy, distal pancreatectomy, and total pancreatectomy in 108 cases (60.7\%), 60 cases (33.7\%), and 10 cases (5.6\%), respectively. R0 and R1 resection were performed in 159 cases $(89.3 \%)$ and 19 cases $(10.7 \%)$, respectively. The median observation period was 22.3 months (8.0-147.2 months). The median overall survival was 30.6 months (95\% CI 25.5-35.7), while the 5-year survival rate was $25.9 \%$. The median recurrence-free survival (RFS) was 13.3 months (95\% CI $10.7-15.9)$, and the 3-year RFS rate was $28.9 \%$. Early recurrence was observed in 45 cases $(25.2 \%)$. The sites of early recurrence included the liver, lungs, peritoneum, distant lymph nodes, and local areas after resection in 26 cases $(57.8 \%), 2$ cases $(4.4 \%), 14$ cases $(31.3 \%), 10$ cases $(22.2 \%)$, and 12 cases $(26.7 \%)$, respectively. Three-year survival rate, 5-year survival rate, and median survival time for the non-ER group were $57.6 \%, 35.5 \%$, and 40.0 months (95\% CT, 29.6-50.5), respectively, whereas those for the early recurrence group were $7.1 \%, 0.0 \%$, and 10.9 months (95\% CI 7.8-14.0), respectively. Significant differences in the OS were observed between the two groups $(p<0.001$, Fig. 1).

\section{Identification of Preoperative Factors Associated with Early Recurrence}

We analyzed preoperative factors associated with early recurrence and found significantly higher levels of preoperative serum CA19-9 (1926.3 versus $292.1 \mathrm{U} / \mathrm{mL}$; $p=0.03)$ and preoperative serum SPan-1 (673.1 versus $108.6 \mathrm{U} / \mathrm{mL} ; p=0.006$ ) in the ER group. It was also revealed that diameter of the tumor on CT was significantly larger (39.4 versus $25.1 \mathrm{~mm} ; p=0.01$ ). Serum DUPAN-2 levels tended to be higher in the ER group (1029.7 versus $526.8 \mathrm{U} / \mathrm{mL} ; \quad p=0.07)$ in the ER group. Other

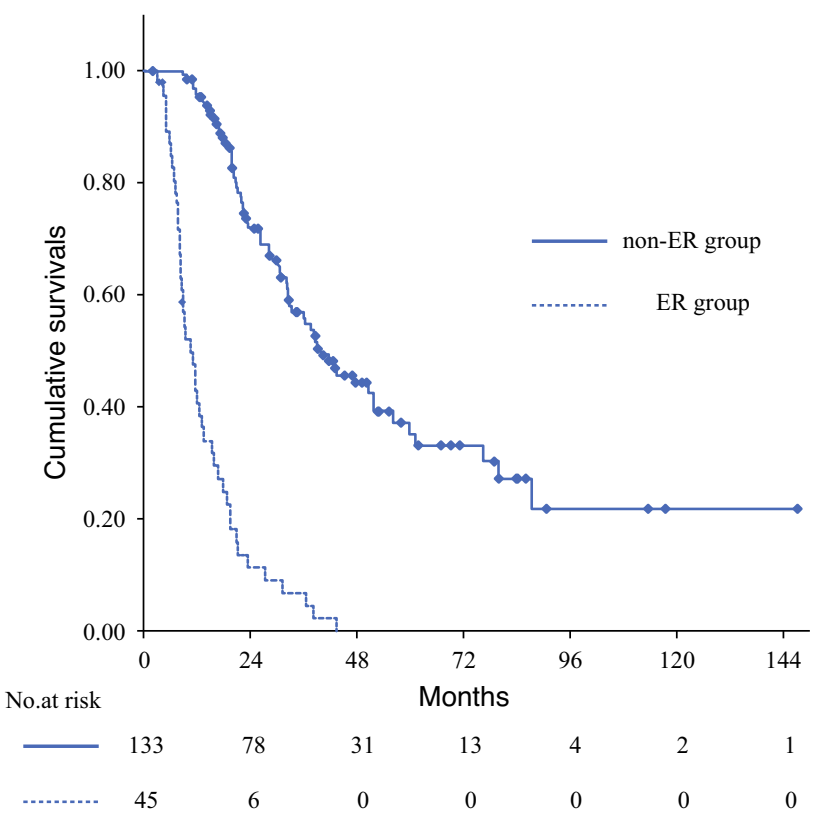

FIG. 1 Comparison of survivals between ER and non-ER groups. On survival analysis by the Kaplan-Meier curve, the 3-year survival rate, 5 -year survival rate, and median survival time of the early recurrence group (solid line) were $7.1 \%, 0.0 \%$, and 10.9 months $(95 \% \mathrm{CI}$ 7.8-14.0), respectively, while the non-early recurrence group (dotted line) had 3-year survival rate, 5-year survival rate, and median survival time of $57.6 \%, 35.5 \%$, and 40.0 months (95\% CI 29.6-50.5), respectively. Log-rank test showed a significant difference in the survival curves between the two groups $(p<0.001)$

preoperative factors such as treatment periods, comorbidities, preoperative nutritional indicators, tumor localization, and max value of standardized uptake value (SUVmax) on PET-CT did not show significant intergroup differences (Table 2). In our institute, gemcitabine/nabpaclitaxel and FOLFIRINOX have been available for PDAC treatment since 2015. Therefore, to assess the impact of effective chemotherapy, we divided the treatment period into early (2005-2014) and late periods (2015-2019) to assess the early recurrence rate. The early recurrence rates in the early and late periods were $30.8 \%$ (24/78) and $21.0 \%$ (21/100), respectively, with no significant difference between the periods $(p=0.14)$.

\section{Identification of Cutoff Values of Serum Tumor Markers and Tumor Diameter for Predicting Early Recurrence}

ROC analysis of the factors associated with early recurrence presented in Table 2 was performed to set their cutoff values for early recurrence prediction (Table 3 ). The cutoff values of preoperative CA19-9, SPan-1, and CTderived tumor diameter were $133 \mathrm{U} / \mathrm{mL}$ [area under curve (AUC) $0.688(0.592-0.783) ; p<0.001$ ], $78.2 \mathrm{U} / \mathrm{mL}$ [AUC $0.721(0.633-0.809) ; p<0.001$ ], and $23 \mathrm{~mm}$ [AUC 0.629 $(0.535-0.723) ; p=0.007]$, respectively. The cutoff values 
TABLE 2 Comparison of the predicting factors for early recurrence between ER and non-ER groups
TABLE 3 ROC curve analysis for predicting early recurrence

\begin{tabular}{llll}
\hline Parameter & ER group & Non-ER group & $p$ \\
\hline Number of patients & 45 & 133 & \\
Age & $69.3 \pm 7.6$ & $69.0 \pm 7.7$ & 0.75 \\
Gender (female/male) & $21 / 24$ & $65 / 68$ & 0.80 \\
Period (2005-2014/2015-2019) & $23 / 22$ & $55 / 78$ & 0.25 \\
BMI & $22.8 \pm 2.9$ & $22.5 \pm 3.7$ & 0.63 \\
ASA score (1,2/3 $\leq$ ) & $41 / 4$ & $118 / 15$ & 0.65 \\
Diabetes mellitus & $11(24.4 \%)$ & $44(33.0 \%)$ & 0.28 \\
Chronic pancreatitis & $1(2.2 \%)$ & $6(4.5 \%)$ & 0.49 \\
Modified GPS (0/1/2) & $37 / 5 / 3$ & $102 / 26 / 5$ & 0.34 \\
NLR & $2.8 \pm 1.5$ & $2.7 \pm 1.9$ & 0.78 \\
PNI & $44.9 \pm 6.45$ & $45.3 \pm 6.2$ & 0.70 \\
Preoperative CA19-9 (U/mL) & $1926.3 \pm 8631.1$ & $292.1 \pm 963.1$ & 0.03 \\
Preoperative SPan-1 (U/mL) & $673.1 \pm 2270.1$ & $108.6 \pm 221.4$ & 0.006 \\
Preoperative DUPAN-2 (U/mL) & $1029.7 \pm 2939.8$ & $526.8 \pm 1247.7$ & 0.07 \\
SUVmax of the tumor on PET-CT & $5.5 \pm 2.3$ & $5.5 \pm 3.4$ & 0.93 \\
Tumor location (Ph/Pbt) & $33 / 18$ & $94 / 48$ & 0.85 \\
Diameter of the pancreatic tumor on CT & $39.4 \pm 10.1$ & $25.1 \pm 9.5$ & 0.01 \\
\hline ER
\end{tabular}

$E R$ early recurrence, Non-ER no recurrence within 6 months after resection, BMI body mass index, Age age at diagnosis, ASA-PS American Society of Anesthesiologists physical status, GPS Glasgow Performance Status, $N L R$ neutrophil-to-lymphocyte ratio, $P N I$ prognostic nutritional index, CA19-9 carbohydrate antigen 19-9, Span-1 s-pancreas-1 antigen, DUPAN-2 Duke pancreas-2 antigen, SUVmax max value of standardized uptake value, $P E T$ positron emission tomography, $C T$ computed tomography, $P h$ pancreatic head, $P b t$ pancreatic body and tail

\begin{tabular}{lllc}
\hline Parameters & Cutoff value & AUC & $p$ \\
\hline Preoperative CA19-9 & $133 \mathrm{U} / \mathrm{mL}$ & $0.688(0.592-0.783)$ & $<0.001$ \\
Preoperative SPan-1 & $78.2 \mathrm{U} / \mathrm{mL}$ & $0.721(0.633-0.809)$ & $<0.001$ \\
Preoperative DUPAN-2 & $126 \mathrm{U} / \mathrm{mL}$ & $0.594(0.499-0.670)$ & 0.052 \\
SUV max on PET-CT & 4.5 & $0.522(0.490-0.754)$ & 0.07 \\
Diameter of the tumor on CT & $23.0 \mathrm{~mm}$ & $0.629(0.535-0.723)$ & 0.007 \\
\hline
\end{tabular}

$A U C$ area under the curve, CA19-9 carbohydrate antigen 19-9, Span-1 s-pancreas-1 antigen, DUPAN-2 Duke pancreas-2 antigen, SUVmax max value of standardized uptake value, PET positron emission tomography, $C T$ computed tomography for preoperative serum DUPAN-2 and SUVmax on PETCT were $126 \mathrm{U} / \mathrm{mL}$ [AUC 0.594 (0.499-0.670); $p=0.052$ ] and 4.5 [AUC $0.522(0.490-0.754) ; p=0.07$ ]. However, the two parameters could not be set as a significant cutoff values for predicting early recurrence.

\section{Stratification of Survival Time by Early Recurrence Prediction Score}

The scoring system for predicting early recurrence was created using the cutoff values of preoperative serum CA19-9 (133 U/mL) and SPan-1 (78.2 U/mL), and preoperative CT-derived tumor diameter $(23 \mathrm{~mm})$. When the parameter exceeded the cutoff level, 1 point was given, and the total score of the three factors was defined as the early recurrence predictive score. Scores of $0,1,2$, and 3 points were found in 46 cases, 62 cases, 39 cases, and 31 cases, respectively. The comparison of RFS and OS between the prediction scores showed that the median RFS time in cases with scores $0,1,2$, and 3 was 24.4 months $(95 \% \mathrm{CI}$ -0.23-49.1), 16.5 months (95\% CI 1.07-22.3), $12.1(95 \%$ CI 5.56-18.7), and 5.5 months (95\% CI 3.02-7.80), respectively. The median OS was 51.8 months $(95 \% \mathrm{CI}$ 18.0-85.6), 30.2 months (95\% CI 20.5-40.0), 26.2 months (95\% CI 12.0-40.4), and 15.9 months (95\% CI 10.4-21.4) in cases with scores of $0,1,2$, and 3, respectively. In the Kaplan-Meier survival analysis, both the RFS time and the OS time were significantly stratified by prediction scores $(p<0.001$, Fig. 2). 


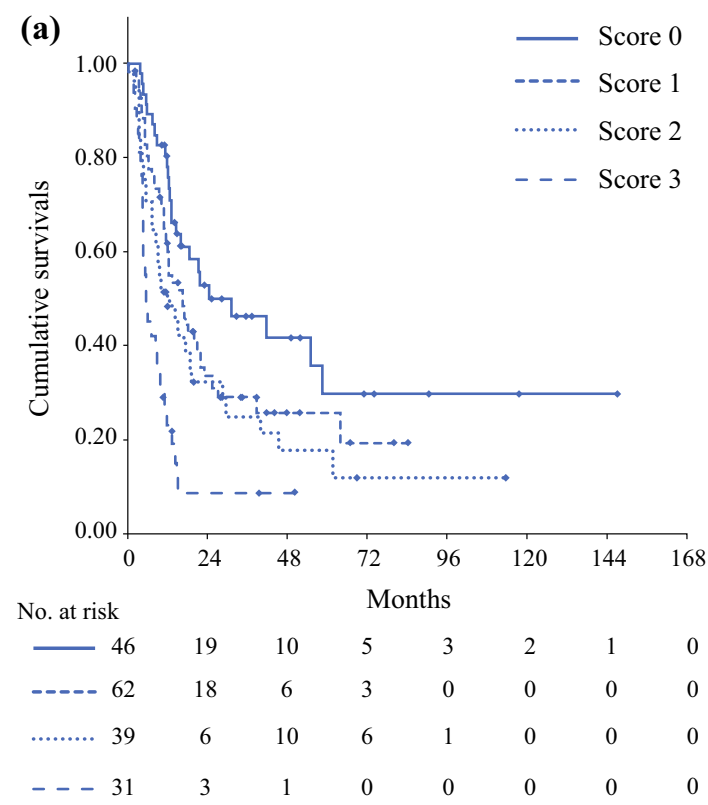

FIG. 2 Stratification of survivals by the early recurrence prediction scores. Survival analysis using the Kaplan-Meier curve was performed stratified by early recurrence prediction scores. A Recurrence-free survival (mRFS) time for cases with scores of 0 , $1,2$, and 3 were 24.4 months (95\% CI $0.2-49.1), 16.5$ months $(95 \%$ CI 10.7-22.4), 10.2 months (95\% CI 4.0-16.5), and 5.5 months (95\%

\section{Univariate and Multivariate Analysis of Factors Predicting Early Recurrence}

Univariate and multivariate analysis using the perioperative factors were performed to identify the independent factors associated with early recurrence (Table 4). On univariate analysis, early recurrence prediction score of 3 points $(p<0.001)$, postoperative complications (ClavienDindo $>$ grade II, $p=0.01$ ), pancreatic fistula (grades B and $\mathrm{C}, p=0.04)$, lymphatic vessel invasion $(p=0.02)$, lymph node metastasis $(p=0.02)$, and vein invasion $(p=0.006)$ were associated with early recurrence. R1 resection $(p=0.05)$ and no postoperative adjuvant chemotherapy $(p=0.07)$ tended to be related with early recurrence; however, they were not significant. Furthermore, multivariate analysis showed that early recurrence prediction score of 3 [odds ratio (OR), 4.63; 95\% CI $11.82-11.78, p=0.0013$ ] and $\mathrm{R} 1$ resection (OR 3.20; 95\% CI $1.01-10.17, p=0.049)$ were independently associated with recurrence within 6 months after curative resection for PDAC.

\section{Recurrence Patterns in the Early Recurrence Group}

The characteristics of the recurrence site in the two groups were examined (Table 5). It was shown that liver and peritoneal metastases were significantly more common

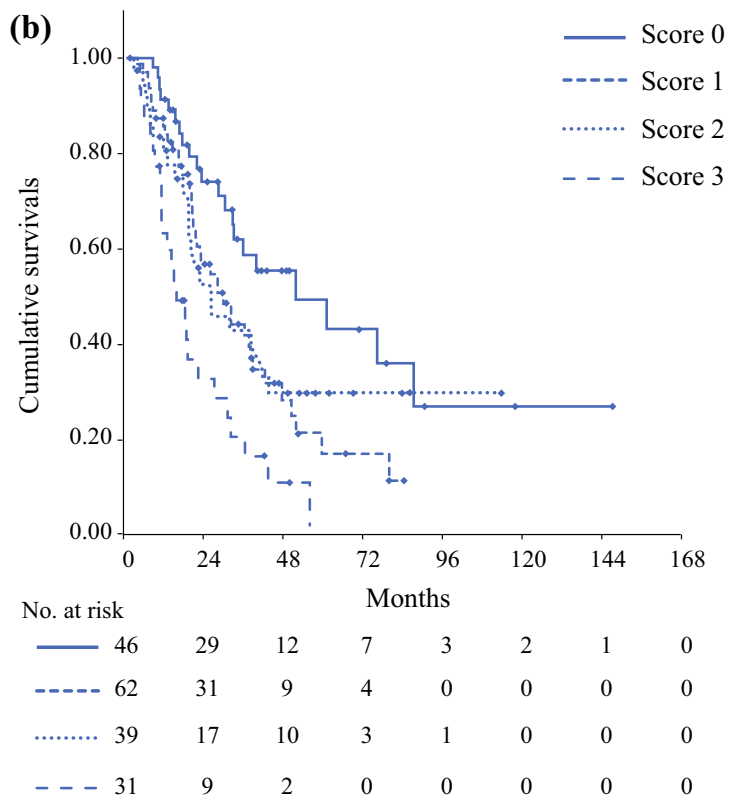

CI 3.0-8.0), respectively, showing a significant difference in the logrank test $(p<0.001)$. B The median OS time of scores $0,1,2$, and 3 was 51.8 months (95\% CI 18.0-85.6), 32.3 months (95\% CI 21.8-42.8), 23.1 months (95\% CI 14.9-31.3), and 15.9 months (95\% CI 10.4-21.4), respectively, also showing a significant difference in the log-rank test $(p<0.001)$

in the ER than in the non-ER group (liver: $54.9 \%$ versus $47.1 \%, p<0.001$; peritoneum: $33.3 \%$ versus $12.0 \%$, $p<0.001)$. However, there were no significant intergroup differences in frequency of recurrence in the lungs, distant lymph nodes, and locoregional site.

\section{DISCUSSION}

In this study, we established early postoperative recurrence prediction scores based on preoperative serum CA199 and SPan-1 levels, as well as preoperative tumor diameter in 178 patients with resectable PDAC who had undergone radical resection. We showed that it was possible to stratify postoperative RFS and OS depending on the predicted score. We also demonstrated that the median RFS time for patients with the prediction score of 3 (preoperative CA19-9 level above $133 \mathrm{U} / \mathrm{mL}$, SPan-1 level above $78.2 \mathrm{U} / \mathrm{mL}$, and CT tumor diameter above $23 \mathrm{~mm}$ ) was 5.5 months. Furthermore, multivariate analysis revealed that a score of 3 was the independent factor associated with recurrence within 6 months after surgery. With respect to the recurrence pattern, the ER group more often had liver metastasis and peritoneal dissemination compared with the non-ER group. In patients with a prediction score of 0 , the median RFS and the median OS time were 24.4 and 51.8 months, respectively, which indicated notably better prognosis. The early recurrence prediction 
TABLE 4 Uni- and multivariate analysis of the predicting factors for early recurrence

\begin{tabular}{|c|c|c|c|c|c|c|}
\hline \multirow[t]{2}{*}{ Parameters } & \multicolumn{3}{|c|}{ Univariate analysis } & \multicolumn{3}{|c|}{ Multivariate analysis } \\
\hline & ER group & Non-ER group & $p$ & Odds ratio & $95 \% \mathrm{CI}$ & $p$ \\
\hline Number of patients & 45 & 133 & & & & \\
\hline Age $(75$ years $<)$ & $11(24.4 \%)$ & $32(24.0 \%)$ & 0.94 & & & \\
\hline Gender (male) & $24(53.3 \%)$ & $68(51.1 \%)$ & 0.80 & & & \\
\hline BMI $(25<)$ & $22.8 \pm 2.9$ & $22.5 \pm 3.7$ & 0.63 & & & \\
\hline ASA-PS $(3 \leq)$ & $4(8.9 \%)$ & $15(11.3 \%)$ & 0.65 & & & \\
\hline Diabetes mellitus & $11(24.4 \%)$ & $44(33.0 \%)$ & 0.28 & & & \\
\hline Chronic pancreatitis & $1(2.2 \%)$ & $6(4.5 \%)$ & 0.49 & & & \\
\hline Modified GPS (2) & $3(6.7 \%)$ & $5(3.8 \%)$ & 0.45 & & & \\
\hline ER predictive score (3) & $18(40.0 \%)$ & $14(10.5 \%)$ & $<0.001$ & 4.63 & $1.82-11.78$ & 0.0013 \\
\hline Tumor location $(\mathrm{Ph})$ & $33(73.3 \%)$ & $94(70.7 \%)$ & 0.85 & & & \\
\hline Blood loss $(1000 \mathrm{ml}<)$ & $20(44.4 \%)$ & $43(32.3 \%)$ & 0.052 & & & \\
\hline Operative time $(360 \min <)$ & $16(35.6 \%)$ & $44(33.1 \%)$ & 0.88 & & & \\
\hline PV/SMV resection $(+)$ & $9(20.0 \%)$ & $21(15.8 \%)$ & 0.69 & & & \\
\hline Transfusion $(+)$ & $12(26.7 \%)$ & $32(24.1 \%)$ & 0.13 & & & \\
\hline PV/SMV invasion $(+)$ & $12(26.7 \%)$ & $25(18.8 \%)$ & 0.26 & & & \\
\hline Overall morbidity (Clavien-Dindo $>$ II) & $15(33.3 \%)$ & $19(14.3 \%)$ & 0.003 & 1.80 & $0.32-10.1$ & 0.51 \\
\hline PF (Grade B, C) & $11(24.4 \%)$ & $14(10.5 \%)$ & 0.018 & 2.60 & $0.37-18.02$ & 0.33 \\
\hline Lymphatic invasion $(+)$ & $37(82.2 \%)$ & $81(60.9 \%)$ & 0.006 & 1.75 & $0.61-5.06$ & 0.30 \\
\hline Venous invasion $(+)$ & $36(80.0 \%)$ & $85(63.9 \%)$ & 0.03 & 2.16 & $0.78-5.92$ & 0.14 \\
\hline Neural invasion $(+)$ & $39(86.7 \%)$ & $103(77.4 \%)$ & 0.14 & & & \\
\hline Lymph node metastasis $(+)$ & $34(75.6 \%)$ & $74(55.6 \%)$ & 0.02 & 1.55 & $0.59-4.10$ & 0.37 \\
\hline $\mathrm{R} 1$ resection & $8(17.8)$ & $10(7.5)$ & 0.051 & 3.20 & $1.01-10.17$ & 0.049 \\
\hline Adjuvant chemotherapy $(-)$ & $13(28.9 \%)$ & $22(16.5 \%)$ & 0.07 & 2.12 & $0.76-2.06$ & 0.15 \\
\hline
\end{tabular}

$E R$ early recurrence, Non-ER no recurrence within 6 months after resection, 95\% CI 95\% confidence interval, Age age at diagnosis, BMI body mass index, ASA-PS American Society of Anesthesiologists physical status, GPS Glasgow Performance Status, $P h$ pancreatic head, $P V$ portal vein, $S M V$ superior mesenteric vein, $P F$ pancreatic fistula

TABLE 5 Comparison of recurrence sites between ER and non-ER groups

\begin{tabular}{lclc}
\hline Recurrence site & ER group & Non-ER group & $p$ \\
\hline Liver & $26(57.8 \%)$ & $22(16.5 \%)$ & $<0.001$ \\
Lung & $2(4.4 \%)$ & $19(14.3 \%)$ & 0.08 \\
Peritoneum & $14(31.1 \%)$ & $15(11.3 \%)$ & 0.002 \\
Distant lymph node & $10(22.2 \%)$ & $26(19.5 \%)$ & 0.70 \\
Locoregional site & $12(26.7 \%)$ & $25(18.8 \%)$ & 0.26
\end{tabular}

$E R$ early recurrence, Non-ER no recurrence within 6 months after resection

score was able to stratify the prognosis of resectable PDAC and may be extremely useful for treatment decisionmaking.

The definition of early recurrence after surgery for PDAC varied among previous reports, from 6 months ${ }^{30,31}$ and 8 months, ${ }^{32}$ to 12 months ${ }^{33}$ after surgery. Groot et al. ${ }^{18}$ classified PDAC patients on the basis of the postoperative recurrence time monthly from within 3 months to within 20 months, and evaluated the difference in prognosis between early and late recurrence focusing on the $p$-value of the survival curve analysis. They showed that the cutoff value for the early recurrence time, which showed the greatest difference in prognosis, was set 12 months after surgery. ${ }^{18}$ They also reported that adjuvant therapy was one of the independent factors that reduced the risk of early recurrence. ${ }^{18}$ Postoperative adjuvant chemotherapy is generally administered for 6 months, ${ }^{6,8,34,35}$ and completion of adjuvant chemotherapy has been reported to be a key factor of long-term survival. ${ }^{36,37}$ However, if recurrence occurred within 6 months after surgery, long-term survival would not be expected because the postoperative adjuvant chemotherapy could not be completed. To identify such cases with extremely malignant tumor behavior, in this study we defined early recurrence as PDAC recurrence within 6 months after surgery. 
CA19-9 antigen is a sugar chain antigen derived from cell membrane glycolipid expressed in colorectal cancer cells, which was first reported by Koprowski. ${ }^{38}$ Since then, many investigators have reported that the antigen was expressed not only in colorectal cancer but also in other gastrointestinal cancers such as PDAC and cholangiocarcinoma. $^{39,40}$ In PDAC, serum CA19-9 levels have been reported to reflect the prognosis of PDAC, ${ }^{11,41,42}$ and the link between cutoff values of preoperative serum CA19-9 levels and early postoperative recurrence and poor prognosis was also investigated. ${ }^{20,30,43-47}$ On the other hand, SPan-1 is a cancer-related antigen recognized by a monoclonal antibody prepared using the human PDAC cell line SW1990 as an immune antigen, and it is a high-molecularweight mucin-like protein. ${ }^{48}$ Although the sensitivity of SPan-1 for PDAC diagnosis is equivalent to that of CA199, SPan-1 is a distinguishing antigen that is synthesized even in Lewis-negative patients. ${ }^{49}$ Similar to CA19-9, SPan-1 is a tumor marker that reflects the prognosis of PDAC, ${ }^{50}$ and it has been reported as a risk factor for recurrence within 6 months after surgery. ${ }^{44,51}$ In addition, the usefulness of immunonutrient indicators $\mathrm{NLR}^{10}$ and $\mathrm{mGPS}^{31}$ as factors related to early recurrence has also been reported. Furthermore, stratification of the prognosis after curative surgery using the factors related to early recurrence has been reported in previous studies. ${ }^{19,20,31,45,52,53}$ In our study, the prediction scoring system using preoperative serum CA19-9, SPan-1, and tumor diameter on CT could stratify the survival time of each score. In addition, median RFS and OS in the ERP score 3 groups were estimated to be 5.5 and 15.9 months, respectively, and this group was considered the highly aggressive PDAC group.

In the present study, CA19-9, SPan-1, and the tumor diameter on CT were also identified as important factors for predicting the early postoperative recurrence of PDAC. CA19-9, SPan-1, and the tumor diameter on CT are factors that indicate the malignant potential of PDAC, and they can be assessed prior to initiating treatment. The combination of these factors may be highly significant for predicting and classifying aggressive PDAC with higher malignant potential before initiating treatment. Matsumoto et al. ${ }^{31}$ conducted a multicenter study and reported that CA19-9 levels, tumor diameter $>30 \mathrm{~mm}$, and a modified Glasgow Prognostic Score of 2 were the independent factors associated early recurrence within 6 months after surgery in patients with R-PDAC. They also demonstrated that the combination of these factors could stratify patient prognosis. In addition, Isaji et al. ${ }^{13}$ proposed a modified definition and criteria for borderline resectable (BR)PDAC, including CA19-9 > 500 U/mL, lymph node metastasis confirmed by biopsy or PET-CT, and an Eastern Cooperative Oncology Group Performance Status (ECOG-
PS) $>2$. Thus, evaluating malignant potential using the preoperative CA19-9 level and other preoperative factors is extremely useful for formulating a treatment plan.

CA19-9 is a tumor marker that recognizes an epitope of the sialyl Lewis A antigen. ${ }^{54,55}$ It is also known that DUPAN-2 recognizes the antigenic epitope of sialyl Lewis $\mathrm{C}$, which is a precursor of sialyl Lewis A antigen. ${ }^{54}$ Conversely, some details about the epitope recognized by SPan-1 are unclear, but it is believed that the epitope is similar, but not identical, to that of CA19-9. It has also been reported that SPan-1 recognizes the CA19-9 epitope and some of the antigenic epitopes of sialyl Lewis $\mathrm{C}$ recognized by DUPAN-2. ${ }^{49}$ When the Lewis blood group is negative, the Lewis antigen gene enzyme that synthesizes the sialyl Lewis A from the sialyl Lewis $\mathrm{C}$ is deficient. As a result, Lewis-negative PDAC is negative for CA19-9. ${ }^{56}$ However, DUPAN-2 and SPan-1 are unique in that they recognize the antigenic epitope of sialyl Lewis $\mathrm{C}$, which is a precursor of the antigenic epitope of sialyl Lewis A. Therefore, they can be evaluated even in patients with Lewis-negative PDAC. Regarding the evaluation of the malignant potential of PDAC, some reports found that the combination of CA19-9 and SPan-1 could be used to evaluate disease activity during chemotherapy for PDAC ${ }^{57}$ and that the combination of CA19-9 and DUPAN-2 could be used to estimate the prognosis of PDAC. ${ }^{58}$ Similarly, the preoperative tumor marker index using the cutoff values of CA19-9, CEA, DUPAN-2, and SPan-1 was useful for predicting the prognosis of PDAC. ${ }^{59}$ As described previously, although SPan-1 and DUPAN-2 could not be biomarkers beyond CA19-9 on their own, when combined with CA19-9, they might act in a complementary manner to enable a more sensitive classification of the malignant potential of PDAC. Although DUPAN-2 was not identified as a predictor of early recurrence in this study, it is considered an important factor in the assessment of malignant potential including early recurrence.

In addition to CA19-9 and SPan-1 levels, the tumor diameter on CT was also a significant factor predictive of early recurrence. Tumor diameter is an imaging parameter associated with major vascular invasion. As tumor size increases, the likelihood of vascular invasion such as portal vein or superior mesenteric artery invasion increases, and vascular invasion is consequently associated with potential multi-organ metastasis via blood vessels. ${ }^{60}$ However, in this study, portal vein resection and histological portal vein invasion were not associated with early recurrence. Therefore, tumor size might be an indicator of malignant potential, separate from vascular invasion. Tumor diameter is an imaging parameter that is not strictly a biomarker. However, the aforementioned findings suggest that tumor diameter could be a biomarker for predicting early recurrence in patients with R-PDAC. These results suggest that 
malignant potential can be evaluated in a complementary manner by combining multiple tumor markers and biomarkers, such as tumor size, and that early recurrence of PDAC within 6 months after surgery can be predicted more sensitively. The ability to predict patients at risk of early recurrence within 6 months before initiating treatment is considered extremely meaningful in terms of avoiding unnecessary surgery.

In our study, significantly high rates of no postoperative adjuvant chemotherapy, positive margins, and regional lymph node metastasis were observed in the ER group. These factors may indicate the malignant potential leading to ER in PDAC. However, they can be mitigated by NAT. The efficacy of postoperative adjuvant chemotherapy has been widely reported. 6,7,61 Adjuvant chemotherapy aims to control local microscopic tumor cells and circulating tumor cells after surgery, and ultimately to improve the postoperative recurrence and survival rates. ${ }^{62}$ However, several problems have been pointed out, such as the inability to introduce or complete postoperative adjuvant chemotherapy owing to delayed recovery from postoperative complications. ${ }^{63}$ Our study also showed that no postoperative adjuvant chemotherapy tended to be associated with ER. Alternatively, NAT can effectively control tumors by introducing systemic treatment when the patient is in the best state. Therefore, for patients with high malignant potential, as indicated by the ERP score, a much longer NAT can be employed for sufficient systemic control. With regard to positive margin, this is an independent poor prognostic factor and an early recurrence-related factor in PDAC. ${ }^{64,65}$ Accurately identifying the extent of PDAC may be difficult, even if it is considered resectable on imaging. In such cases, resection margins are more likely to be positive; consequently, sufficient prognosis would not be provided. NAT is likely to result in tumor shrinkage, which is meaningful in preventing positive resection margins. Lastly, regional lymph node metastasis is also a poor prognostic factor in PDAC. However, the contribution of lymph node dissection to prolonged prognosis in lymph node-positive PDAC is unclear. ${ }^{66-68}$ If regional lymph node metastases are controlled by NAT, ER can be prevented and postoperative survival can be prolonged.

Recently, the usefulness of NAT not only for borderline resectable PDAC but also for resectable PDAC has been reported. $^{22,23,69}$ In a multicenter randomized controlled trial, Motoi et al. compared preoperative chemotherapy with GEM and S-1 with upfront surgery (UpS) for resectable PDAC. ${ }^{70}$ At the ASCO-GI meeting in 2019, results of the randomized control trial (RCT) were reported. The results showed that preoperative NAT with GEM/ S-1 showed a significantly higher median survival time (MST) compared with the UpS group. ${ }^{71}$ On the other hand, the disadvantages of NAT have also been reported. ${ }^{72}$ In a meta-analysis of NAT for resectable PDAC, Zhan et al. reported that NAT had no apparent effect on prolonging survival. They suggested that NAT should be carefully indicated for resectable PDAC because of the possibility of disease progression during NAT and the need for more invasive diagnostic techniques for pretreatment diagnosis. $^{72}$ In 2020, a multicenter phase III trial comparing preoperative chemoradiotherapy (CRT) for resectable PDAC with $\mathrm{UpS}$ reported that CRTs for borderline resectable and resectable PDAC had no effect on survival prolongation. ${ }^{73}$ They investigated the usefulness of preoperative chemoradiotherapy (CRT) for resectable and BR-PDAC. In the intention-to-treat analysis, the preoperative CRT group had significantly better disease-free survival and locoregional failure-free survival; however, there was no significant difference in OS between the two groups. Furthermore, subgroup analysis did not reveal a contribution of preoperative CRT to OS in patients with R-PDAC. However, in this study, the chemotherapy used during preoperative CRT was gemcitabine, which was not as potent as gemcitabine/nab-paclitaxel or FOFIRINOX. Further clinical studies on preoperative CRT for $\mathrm{R}-\mathrm{PDAC}$ with such agents are expected to clarify this issue.

Concerning NAT for R-PDAC, it remains controversial because there was not sufficient evidence to demonstrate the prognostic advantages. One of the reasons is that, even if the PDAC was considered to be resectable based on the CT image, it includes a group of cases with an extremely poor prognosis that recur within 6 months after the radical resection. In our study, in PDAC classified as resectable on the CT images, we found that liver metastasis and peritoneal dissemination were significantly more common in the group that recurred within 6 months after radical resection. It is possible that such groups may have potentially distant metastases at the time of surgery, or may have extremely high malignancy that cannot be controlled by surgery. Even if several courses of NAT were introduced in these groups with extremely poor prognosis, it would be difficult to control such tumor dynamics and prolong postoperative survival. For such PDACs with high malignant potential, a long-term NAT for sufficient tumor control might be indicated to improve the survivals.

Several reports have been published on the prediction of ER of PDAC and treatment selection based on the prediction. Oba et al. $^{74}$ defined radiologically occult metastatic pancreatic cancer (ROMPC) as distant metastases revealed during surgery or recurrence within 6 months after surgery. They stated that ROMPC had a significantly lower survival rate than non-ROMPC, and upfront surgery for ROPMC is not beneficial. They also reported that the risk of ROMPC can be predicted if both CA19-9 level of $>300 \mathrm{U} / \mathrm{mL}$ and tumor diameter of $>30 \mathrm{~mm}$ were met. They suggested that ROMPC should 
be predicted preoperatively to apply neoadjuvant chemotherapy. Kurahara et al. ${ }^{45}$ studied the ER of radiographically R-PDAC. They reported that CA19-9 level of $>85 \mathrm{U} / \mathrm{mL}$ and a p53 expression rate of $0 \%$ or $>80 \%$ in tumor cells were independent risk factors for ER. Patients who presented at least one of these factors were defined as high risk, and their survival rates were compared with those of NAC patients. NAC had a survival benefit in the highrisk group. Takahashi et al. ${ }^{75}$ classified radiographically R-PDAC with CA19-9 levels of $>120 \mathrm{U} / \mathrm{mL}$ as biologically BR-PDAC (bBR-PDAC). Although the survival rate of bBR-PDAC was comparable to that of anatomically BRPDAC, the prognosis was significantly improved when CA19-9 was normalized by preoperative CRT. However, in the same report, they found that CA19-9 levels did not normalize after preoperative CRT in approximately $50 \%$ of cases, which were associated with a significantly higher rate of distant recurrences. Thus, predicting early recurrence by using preoperative factors such as CA19-9 and applying NAT may improve the prognosis of the early recurrence group. However, further clinical studies with a higher level of evidence should be conducted.

In our study, the median RFS was only 5.5 months for individuals with an early recurrence prediction score of 3 points (e.g., with CA19-9 > $133 \mathrm{U} / \mathrm{mL}$, SPan-1 > $78.2 \mathrm{U} /$ $\mathrm{mL}$, and CT tumor diameter $>23 \mathrm{~mm}$ ). Furthermore, their median OS was 15.9 months, which was an extremely poor prognosis in spite of R-PDAC. Such poor prognosis groups could be considered biologically unresectable PDAC and, therefore, it seems that the treatment strategy aiming at conversion surgery ${ }^{76}$ after sufficient tumor control by chemotherapy or total neoadjuvant therapy $(\mathrm{TNT})^{77}$ should be applied. TNT is a reported preoperative treatment for borderline resectable and locally advanced unresectable PDAC that combines preoperative intensive chemotherapy and chemoradiotherapy. The treatment concept of TNT is combining systemic control for occult micro-metastasis with local control for negative resection margins. Truty et al. ${ }^{77}$ have reported the usefulness of TNT for BR/LA PDAC, suggesting that long-term chemotherapy of more than six cycles, negative CA19-9 after chemotherapy, and sufficient pathological response were independent factors of long-term survival. Although TNT is a therapeutic strategy for BR/LA PDAC, it should be indicated in cases with resectable PDAC that are biologically unresectable PDAC, such as those with an early recurrence prediction score of 3 . Therefore, the ERP scoring system may have great significance in identifying patients with R-PDAC and poor prognoses and avoiding unnecessary surgery.

On the other hand, the group classified with ERP score of 0 had a median RFS of 24.4 months, a median OS of 54.8 months, and a 5-year survival rate of $49.2 \%$, which was a notably better prognosis. According to the previous reports $^{71,78-80}$ that investigated the efficacy of NAT for resectable PDAC, MST of NAT group was 17.4-38.2 months, compared with MST in surgery-first group of 14.4-26.4 months. The recent report by Unno et al., ${ }^{71}$ which was a multicenter randomized controlled trial comparing NAT with GEM and S-1 with UpS for resectable PDAC, also reported that MST of NAT group was estimated to 36.7 months (HR, 0.72; $p=0.015$ ). Judging from the long MST of 54.8 months of the prediction score 0 in our study, the prognosis of the group would not be expected to be further improved by NAT. Instead, appropriate upfront surgery and the prompt introduction of adjuvant chemotherapy should be indicated for such a group. Recently, much better prognosis has been reported with adjuvant chemotherapy with FOLFIRINOX. ${ }^{8}$ Therefore, for the group classified as our ERP score of 0 , postoperative complications and malnutrition after radical surgery should be prevented as much as possible, and postoperative adjuvant chemotherapy should be smoothly introduced according to the previous reports. ${ }^{37,81}$

This study had the following limitations. First, this was a retrospective study with a limited number of patients in a single institution. Second, since 5-10\% of Japanese have Lewis-negative blood type, CA19-9 could not be detected in such cases. However, Lewis blood type was not assessed in this study. Third, postoperative adjuvant chemotherapy was not unified, and multiple regimens were used. Fourth, due to the historical background, there were cases in which efficacious chemotherapy for PDAC such as GEM and nabpaclitaxel combination therapy or FOLFIRINOX could not be used for recurrence treatment, and there might be a bias in survival time after recurrence. Fifth, our cases included obstructive jaundice cases, which may have affected the levels of CA19-9 and SPan-1. Sixth, PET-CT and cytology with diagnostic laparoscopy for preoperative distant metastasis evaluation were not performed in all patients. Seventh, our study did not address the optimal method or duration of NAT to control tumor dynamics. When NAT has no obvious effect on patients with high ERP scores, in reality, radical surgery often becomes the only option. Thus, preoperative stratification of prognosis using ERP scores may not be practical in determining the treatment strategy. Finally, in our study, cases with R1 resection were also included. In principle, cancer relapse after R1 resection might be considered progression and not recurrence. The ER group may thus have included two different tumor dynamics: recurrence and progression. Despite these limitations, this study had a strong point that the early recurrence prediction scoring system could estimate an ER group with a poor prognosis, which could help to avoid meaningless surgery. 
In conclusion, for resectable PDAC, an early recurrence prediction scoring system using preoperative serum CA199 level, preoperative serum SPan-1 level, and preoperative CT tumor diameter was effective to predict early recurrence within 6 months after surgery. The system could have great significance in avoiding unnecessary surgery. Furthermore, it could stratify the patients according to their predictive prognosis, which may be useful for determining the adaptation to neoadjuvant therapy for resectable PDAC.

DISCLOSURE There are no conflicts of interest to declare.

OPEN ACCESS This article is licensed under a Creative Commons Attribution 4.0 International License, which permits use, sharing, adaptation, distribution and reproduction in any medium or format, as long as you give appropriate credit to the original author(s) and the source, provide a link to the Creative Commons licence, and indicate if changes were made. The images or other third party material in this article are included in the article's Creative Commons licence, unless indicated otherwise in a credit line to the material. If material is not included in the article's Creative Commons licence and your intended use is not permitted by statutory regulation or exceeds the permitted use, you will need to obtain permission directly from the copyright holder. To view a copy of this licence, visit http://creativecommons. org/licenses/by/4.0/.

\section{REFERENCES}

1. Siegel RL, Miller KD, Jemal A. Cancer statistics, 2019. $C A$ Cancer J Clin. 2019;69(1):7-34.

2. Rahib L, Smith BD, Aizenberg R, Rosenzweig AB, Fleshman JM, Matrisian LM. Projecting cancer incidence and deaths to 2030: the unexpected burden of thyroid, liver, and pancreas cancers in the United States. Cancer Res. 2014;74(11):2913-21.

3. Luo G, Zhang Y, Guo P, Ji H, Xiao Y, Li K. Global patterns and trends in pancreatic cancer incidence: age, period, and birth cohort analysis. Pancreas. 2019;48(2):199-208.

4. McGuigan A, Kelly P, Turkington RC, Jones C, Coleman HG, McCain RS. Pancreatic cancer: a review of clinical diagnosis, epidemiology, treatment and outcomes. World J Gastroenterol. 2018;24(43):4846-61.

5. Aier I, Semwal R, Sharma A, Varadwaj PK. A systematic assessment of statistics, risk factors, and underlying features involved in pancreatic cancer. Cancer Epidemiol. 2019;58:104-10.

6. Uesaka K, Boku N, Fukutomi A, et al. Adjuvant chemotherapy of S-1 versus gemcitabine for resected pancreatic cancer: a phase 3, open-label, randomised, non-inferiority trial (JASPAC 01). Lancet. 2016;388(10041):248-57.

7. Neoptolemos JP, Palmer DH, Ghaneh P, et al. Comparison of adjuvant gemcitabine and capecitabine with gemcitabine monotherapy in patients with resected pancreatic cancer (ESPAC-4): a multicentre, open-label, randomised, phase 3 trial. Lancet. 2017;389(10073):1011-24.

8. Conroy T, Hammel P, Hebbar M, et al. FOLFIRINOX or Gemcitabine as adjuvant therapy for pancreatic cancer. $N$ Engl $\mathrm{J}$ Med. 2018;379(25):2395-406.

9. Bergquist JR, Puig CA, Shubert CR, et al. Carbohydrate antigen 19-9 elevation in anatomically resectable, early stage pancreatic cancer is independently associated with decreased overall survival and an indication for neoadjuvant therapy: a National Cancer Database study. J Am Coll Surg. 2016;223(1):52-65.

10. Kim WJ, Lim TW, Park PJ, Choi SB, Kim WB. Prognostic impact of the combination of the neutrophil-to-lymphocyte ratio and serum carbohydrate antigen 19-9 in patients with pancreas head cancer. ANZ J Surg. 2019;89(7-8):e302-7.

11. Ishido K, Hakamada K, Kimura N, Miura T, Wakiya T. Essential updates 2018/2019: current topics in the surgical treatment of pancreatic ductal adenocarcinoma. Ann Gastroenterol Surg. 2021;5(1):7-23.

12. Kim TH, Han SS, Park SJ, et al. CA 19-9 level as indicator of early distant metastasis and therapeutic selection in resected pancreatic cancer. Int $J$ Radiat Oncol Biol Phys. 2011;81(5):e743-8.

13. Isaji S, Mizuno S, Windsor JA, et al. International consensus on definition and criteria of borderline resectable pancreatic ductal adenocarcinoma 2017. Pancreatology. 2018;18(1):2-11.

14. Goh SK, Gold G, Christophi C, Muralidharan V. Serum carbohydrate antigen 19-9 in pancreatic adenocarcinoma: a mini review for surgeons. ANZ J Surg. 2017;87(12):987-92.

15. Shin SH, Kim SC, Song KB, et al. Chronologic changes in clinical and survival features of pancreatic ductal adenocarcinoma since 2000: a single-center experience with 2,029 patients. Surgery. 2018;164(3):432-42.

16. Nakagawa S, Yamashita YI, Umezaki N, et al. Serum marker score based on prognostic nutrition index, carcinoembryonic antigen, and carbohydrate antigen 19-9 is associated with recurrence for patients undergoing surgery for pancreatic ductal adenocarcinoma. Pancreas. 2018;47(9):1130-4.

17. Lowder CY, Metkus J, Epstein J, et al. Clinical implications of extensive lymph node metastases for resected pancreatic cancer. Ann Surg Oncol. 2018;25(13):4004-11.

18. Groot VP, Gemenetzis G, Blair AB, et al. Defining and predicting early recurrence in 957 patients with resected pancreatic ductal adenocarcinoma. Ann Surg. 2019;269(6):1154-62.

19. Nishio K, Kimura K, Amano R, et al. Preoperative predictors for early recurrence of resectable pancreatic cancer. World J Surg Oncol. 2017;15(1):16.

20. Nakamura T, Asano T, Okamura K, et al. A preoperative prognosticscoring system to predict prognosis for resectable pancreatic cancer: who will benefit from upfront surgery? J Gastrointest Surg. 2019;23(5):990-6.

21. Gillen S, Schuster T, Meyer Zum Büschenfelde C, Friess H, Kleeff J. Preoperative/neoadjuvant therapy in pancreatic cancer: a systematic review and meta-analysis of response and resection percentages. PLoS Med. 2010;7(4):e1000267.

22. Versteijne E, Vogel JA, Besselink MG, et al. Meta-analysis comparing upfront surgery with neoadjuvant treatment in patients with resectable or borderline resectable pancreatic cancer. $\mathrm{Br} \mathrm{J}$ Surg. 2018;105(8):946-58.

23. Ye M, Zhang Q, Chen Y, et al. Neoadjuvant chemotherapy for primary resectable pancreatic cancer: a systematic review and meta-analysis. HPB. 2020. https://doi.org/10.1016/j.hpb.2020.01. 001.

24. Ahmad SA, Duong M, Sohal DPS, et al. Surgical outcome results from SWOG S1505: a randomized clinical trial of mFOLFIRINOX versus Gemcitabine/Nab-paclitaxel for perioperative treatment of resectable pancreatic ductal adenocarcinoma. Ann Surg. 2020;272(3):481-6.

25. Sohal DPS, Duong M, Ahmad SA, et al. Efficacy of perioperative chemotherapy for resectable pancreatic adenocarcinoma: a phase 2 randomized clinical trial. JAMA Oncol. 2021;7(3):421-7.

26. National Comprehensive Cancer Network NCCN clinical practice guidelines in oncology (NCCN guidelines) pancreatic adenocarcinoma version 1. 2020; 
27. Clavien PA, Barkun J, de Oliveira ML, et al. The Clavien-Dindo classification of surgical complications: five-year experience. Ann Surg. 2009;250(2):187-96.

28. Bassi C, Dervenis C, Butturini G, et al. Postoperative pancreatic fistula: an international study group (ISGPF) definition. Surgery. 2005;138(1):8-13.

29. Brierley JD GMWC. UICC: TMN classification of malignant tumors. 8th edn. Wiley-Blackwell; 2017.

30. Sugiura T, Uesaka K, Kanemoto H, et al. Serum CA19-9 is a significant predictor among preoperative parameters for early recurrence after resection of pancreatic adenocarcinoma. J Gastrointest Surg. 2012;16(5):977-85.

31. Matsumoto I, Murakami Y, Shinzeki M, et al. Proposed preoperative risk factors for early recurrence in patients with resectable pancreatic ductal adenocarcinoma after surgical resection: a multi-center retrospective study. Pancreatology. 2015;15(6):674-80.

32. Niedergethmann M, Hildenbrand R, Wostbrock B, et al. High expression of vascular endothelial growth factor predicts early recurrence and poor prognosis after curative resection for ductal adenocarcinoma of the pancreas. Pancreas. 2002;25(2):122-9.

33. Zhai LL, Wu Y, Huang DW, Tang ZG. Increased matrix metalloproteinase-2 expression and reduced tissue factor pathway inhibitor-2 expression correlate with angiogenesis and early postoperative recurrence of pancreatic carcinoma. Am J Transl Res. 2015;7(11):2412-22.

34. Oettle H, Post S, Neuhaus P, et al. Adjuvant chemotherapy with gemcitabine vs observation in patients undergoing curative-intent resection of pancreatic cancer: a randomized controlled trial. JAMA. 2007;297(3):267-77.

35. Neoptolemos JP, Palmer DH, Ghaneh P, et al. Comparison of adjuvant gemcitabine and capecitabine with gemcitabine monotherapy in patients with resected pancreatic cancer (ESPAC-4): a multicentre, open-label, randomised, phase 3 trial. Lancet (London, England). 2017;389(10073):1011-24.

36. Ma SJ, Oladeru OT, Miccio JA, Iovoli AJ, Hermann GM, Singh AK. Association of timing of adjuvant therapy with survival in patients with resected stage I to II pancreatic cancer. JAMA Netw Open. 2019;2(8):e199126.

37. DePeralta DK, Ogami T, Zhou JM, et al. Completion of adjuvant therapy in patients with resected pancreatic cancer. $H P B$. 2020;22(2):241-8.

38. Koprowski H, Steplewski Z, Mitchell K, Herlyn M, Herlyn D, Fuhrer P. Colorectal carcinoma antigens detected by hybridoma antibodies. Somat Cell Genet. 1979;5(6):957-71.

39. Humphris JL, Chang DK, Johns AL, et al. The prognostic and predictive value of serum CA199 in pancreatic cancer. Ann Oncol. 2012;23(7):1713-22.

40. Yamashita S, Passot G, Aloia TA, et al. Prognostic value of carbohydrate antigen 19-9 in patients undergoing resection of biliary tract cancer. Br J Surg. 2017;104(3):267-77.

41. Nakao A, Oshima K, Nomoto S, et al. Clinical usefulness of CA19-9 in pancreatic carcinoma. Sem Surg Oncol. 1998;15(1):15-22.

42. Kimura K, Amano R, Nakata B, et al. Clinical and pathological features of five-year survivors after pancreatectomy for pancreatic adenocarcinoma. World J Surg Oncol. 2014;12:360.

43. Shimizu T, Asakuma M, Tomioka A, et al. Span-1 and CA19-9 as Predictors of early recurrence and lymph node metastasis for patients with invasive pancreatic cancer after pancreatectomy. Am Surg. 2018;84(1):109-13.

44. Suzuki S, Shimoda M, Shimazaki J, et al. Predictive early recurrence factors of preoperative clinicophysiological findings in pancreatic cancer. Eur Surg Res. 2018;59(5-6):329-38.
45. Kurahara H, Maemura K, Mataki Y, et al. A therapeutic strategy for resectable pancreatic cancer based on risk factors of early recurrence. Pancreas. 2018;47(6):753-8.

46. Santucci N, Facy O, Ortega-Deballon P, Lequeu JB, Rat P, Rat P. CA 19-9 predicts resectability of pancreatic cancer even in jaundiced patients. Pancreatology. 2018;18(6):666-70.

47. Herreros-Villanueva M, Ruiz-Rebollo L, Montes M, et al. CA199 capability as predictor of pancreatic cancer resectability in a Spanish cohort. Mol Biol Rep. 2020;47(3):1583-8.

48. Ho JJ, Chung YS, Fujimoto Y, et al. Mucin-like antigens in a human pancreatic cancer cell line identified by murine monoclonal antibodies SPan-1 and YPan-1. Cancer Res. 1988;48(14):3924-31.

49. Kawa S, Tokoo M, Oguchi H, et al. Epitope analysis of SPan-1 and DUPAN-2 using synthesized glycoconjugates sialyllact-Nfucopentaose II and sialyllact-N-tetraose. Pancreas. 1994;9(6):692-7.

50. Kiriyama S, Hayakawa T, Kondo T, et al. Usefulness of a new tumor marker, Span-1, for the diagnosis of pancreatic cancer. Cancer. 1990;65(7):1557-61.

51. Hosokawa Y, Nagakawa Y, Sahara Y, Takishita C, Katsumata K, Tsuchida A. Serum SPan-1 is a significant risk factor for early recurrence of pancreatic cancer after curative resection. Dig Surg. 2017;34(2):125-32.

52. Numata K, Morinaga S, Katayama $Y$, et al. Combining the Glasgow Prognostic Score and serum carbohydrate antigen 19-9 level improves the ability to predict early recurrence in resected pancreatic cancer patients receiving adjuvant Gemcitabine. Anticancer Res. 2016;36(5):2467-74.

53. Izumo W, Higuchi R, Furukawa T, et al. Evaluation of preoperative prognostic factors in patients with resectable pancreatic ductal adenocarcinoma. Scand J Gastroenterol. 2019;54(6):780-6.

54. Kawa S, Oguchi H, Kobayashi T, et al. Elevated serum levels of Dupan-2 in pancreatic cancer patients negative for Lewis blood group phenotype. Br J Cancer. 1991;64(5):899-902.

55. Chen HL. Lewis glyco-epitopes: structure, biosynthesis, and functions. Adv Exp Med Biol. 2011;705:53-80.

56. Tempero MA, Uchida E, Takasaki H, Burnett DA, Steplewski Z, Pour PM. Relationship of carbohydrate antigen 19-9 and Lewis antigens in pancreatic cancer. Cancer Res. 1987;47(20):5501-3.

57. Tsutsumi K, Kawamoto H, Hirao K, et al. Monitoring of CA19-9 and SPan-1 can facilitate the earlier confirmation of progressing pancreatic cancer during chemotherapy. Pancreatology. 2012;12(5):409-16.

58. Shibata K, Iwaki K, Kai S, Ohta M, Kitano S. Increased levels of both carbohydrate antigen 19-9 and duke pancreatic monoclonal antigen type 2 reflect postoperative prognosis in patients with pancreatic carcinoma. Pancreas. 2009;38(6):619-24.

59. Miyata T, Hayashi H, Yamashita YI, et al. Prognostic value of the preoperative tumor marker index in resected pancreatic ductal adenocarcinoma: a retrospective single-institution study. Ann Surg Oncol. 2021;28(3):1572-80.

60. Mierke F, Hempel S, Distler M, et al. Impact of portal vein involvement from pancreatic cancer on metastatic pattern after surgical resection. Ann Surg Oncol. 2016;23(Suppl 5):730-6.

61. Oettle H, Neuhaus P, Hochhaus A, et al. Adjuvant chemotherapy with gemcitabine and long-term outcomes among patients with resected pancreatic cancer: the CONKO-001 randomized trial. JAMA. 2013;310(14):1473-81.

62. Heinrich S, Lang H. Neoadjuvant therapy of pancreatic cancer: definitions and benefits. Int J Mol Sci. 2017;18(8):1622.

63. Merkow RP, Bilimoria KY, Bentrem DJ, et al. National assessment of margin status as a quality indicator after pancreatic cancer surgery. Ann Surg Oncol. 2014;21(4):1067-74. 
64. Ghaneh P, Kleeff J, Halloran CM, et al. The impact of positive resection margins on survival and recurrence following resection and adjuvant chemotherapy for pancreatic ductal adenocarcinoma. Ann Surg. 2019;269(3):520-9.

65. Tummers WS, Groen JV, Sibinga Mulder BG, et al. Impact of resection margin status on recurrence and survival in pancreatic cancer surgery. Br J Surg. 2019;106(8):1055-65.

66. Nimura Y, Nagino M, Takao S, et al. Standard versus extended lymphadenectomy in radical pancreatoduodenectomy for ductal adenocarcinoma of the head of the pancreas: long-term results of a Japanese multicenter randomized controlled trial. J Hepatobiliary Pancreat Sci. 2012;19(3):230-41.

67. Jang JY, Kang MJ, Heo JS, et al. A prospective randomized controlled study comparing outcomes of standard resection and extended resection, including dissection of the nerve plexus and various lymph nodes, in patients with pancreatic head cancer. Ann Surg. 2014;259(4):656-64.

68. Jang JY, Kang JS, Han Y, et al. Long-term outcomes and recurrence patterns of standard versus extended pancreatectomy for pancreatic head cancer: a multicenter prospective randomized controlled study. $J$ Hepatobiliary Pancreat Sci. 2017;24(7):426-33.

69. Bradley A, Van Der Meer R. Neoadjuvant therapy versus upfront surgery for potentially resectable pancreatic cancer: a Markov decision analysis. PLoS One. 2019;14(2):e0212805.

70. Motoi F, Kosuge T, Ueno H, et al. Randomized phase II/III trial of neoadjuvant chemotherapy with gemcitabine and S-1 versus upfront surgery for resectable pancreatic cancer (Prep-02/ JSAP05). Jpn J Clin Oncol. 2019;49(2):190-4.

71. Unno M, Motoi F, Matsuyama Y, et al. Randomized phase II/III trial of neoadjuvant chemotherapy with gemcitabine and S-1 versus upfront surgery for resectable pancreatic cancer (Prep-02/ JSAP-05). J Clin Oncol. 2019;37(suppl 4):abstr 189.

72. Zhan HX, Xu JW, Wu D, et al. Neoadjuvant therapy in pancreatic cancer: a systematic review and meta-analysis of prospective studies. Cancer Med. 2017;6(6):1201-19.

73. Versteijne E, Suker M, Groothuis K, et al. Preoperative chemoradiotherapy versus immediate surgery for resectable and borderline resectable pancreatic cancer: results of the Dutch randomized phase III PREOPANC trial. J Clin Oncol. 2020;38(16):1763-73.
74. Oba A, Inoue Y, Ono Y, et al. Radiologically occult metastatic pancreatic cancer: how can we avoid unbeneficial resection? Langenbeck's Arch Surg. 2020;405(1):35-41.

75. Takahashi H, Yamada D, Asukai K, et al. Clinical implications of the serum CA19-9 level in "biological borderline resectability" and "biological downstaging" in the setting of preoperative chemoradiation therapy for pancreatic cancer. Pancreatology. 2020;20(5):919-28.

76. Satoi S, Yamaue H, Kato K, et al. Role of adjuvant surgery for patients with initially unresectable pancreatic cancer with a longterm favorable response to non-surgical anti-cancer treatments: results of a project study for pancreatic surgery by the Japanese Society of Hepato-Biliary-Pancreatic Surgery. J Hepatobiliary Pancreat Sci. 2013;20(6):590-600.

77. Truty MJ, Kendrick ML, Nagorney DM, et al. Factors predicting response, perioperative outcomes, and survival following total neoadjuvant therapy for borderline/locally advanced pancreatic cancer. Ann Surg. 2019;273(2):341-9.

78. Golcher H, Brunner TB, Witzigmann H, et al. Neoadjuvant chemoradiation therapy with gemcitabine/cisplatin and surgery versus immediate surgery in resectable pancreatic cancer: results of the first prospective randomized phase II trial. Strahlenther Onkol. 2015;191(1):7-16.

79. Casadei R, Di Marco M, Ricci C, et al. Neoadjuvant chemoradiotherapy and surgery versus surgery alone in resectable pancreatic cancer: a single-center prospective, randomized, controlled trial which failed to achieve accrual targets. $J$ Gastrointest Surg. 2015;19(10):1802-12.

80. Reni M, Balzano G, Zanon S, et al. Safety and efficacy of preoperative or postoperative chemotherapy for resectable pancreatic adenocarcinoma (PACT-15): a randomised, open-label, phase 2-3 trial. Lancet Gastroenterol Hepatol. 2018;3(6):413-23.

81. Kim HW, Lee JC, Lee J, Kim JW, Kim J, Hwang JH. Early versus delayed initiation of adjuvant treatment for pancreatic cancer. PLoS One. 2017;12(3):e0173960.

Publisher's Note Springer Nature remains neutral with regard to jurisdictional claims in published maps and institutional affiliations. 